\title{
Prompt fission gamma rays and their angular distributions
}

\author{
Andreas Oberstedt ${ }^{1, \star}$, Robert Billnert ${ }^{2,3}$, Angélique Gatera ${ }^{2}$, Alf Göök ${ }^{2}$, and Stephan Oberstedt ${ }^{2}$ \\ ${ }^{1}$ Extreme Light Infrastructure - Nuclear Physics (ELI-NP) / Horia Hulubei National Institute for Physics and \\ Nuclear Engineering (IFIN-HH), 077125 Bucharest-Magurele, Romania \\ ${ }^{2}$ European Commission, DG Joint Research Centre, Directorate G - Nuclear Safety and Security, Unit G.2 \\ Standards for Nuclear Safety, Security and Safeguards, 2440 Geel, Belgium \\ ${ }^{3}$ Present address: Studsvik Nuclear Environmental AB, 61182 Nyköping, Sweden
}

\begin{abstract}
In a recent measurement of prompt $\gamma$ rays from the spontaneous fission of ${ }^{252} \mathrm{Cf}$ an experimental setup was chosen that allowed the study of angular correlations between $\gamma$ rays and the nuclei from which they were emitted. The observed angular distribution of the prompt fission $\gamma$ rays was then analyzed to estimate the relative contributions from radiation of different multipolarity. From that we hope to obtain more detailed information about the early stage of the de-excitation process of fission fragments, right after prompt neutron emission. First preliminary results are presented below.
\end{abstract}

\section{Introduction}

Nuclear fission is a complex process, which - after almost 80 years since its discovery - is still not fully understood. One field of research is for instance studies of the de-excitation of fission fragments, which in the early stages takes place through the successive emission of prompt neutrons and $\gamma$ rays. For nuclear applications, information about the prompt neutrons is crucial for calculating the reactivity in reactors, while precise knowledge about the prompt $\gamma$ rays is important for the assessment of the decay heat released in the reactor core. Concerning the latter we have contributed in the past years with a number of precise measurements of prompt $\gamma$-ray spectra from the spontaneous [1-3] as well as thermal [4-6] and fast neutron [7, 8] induced fission of various compound systems. In these experiments prompt fission $\gamma$-ray spectra (PFGS) were measured and their characteristics, i.e. average total $\gamma$-ray energy released in prompt fission, mean energy per photon as well as average photon multiplicity, were determined with unprecedented accuracy. This collection of obtained experimental data was also used to study the dependence of PFGS characteristics of mass and atomic number of the fissioning system as well as of its excitation energy [9].

The energy release in nuclear fission is distributed in kinetic and excitation energy of the two fragments. The latter manifests itself in fragment deformation and intrinsic excitation energy. In an early and quite simplified picture, it was assumed that the fragments are first de-excited by the emission of neutrons until the fragments' remaining excitation energy is lower than the neutron binding energy, i.e. on average at about half of it. Only then de-excitation would continue by the emission of $\gamma$ rays, eventually reaching the ground state [10]. These $\gamma$ rays may be divided into two categories, commonly called statistical and discrete $\gamma$ rays, respectively. The first ones originate mainly from electric

\footnotetext{
^e-mail: andreas.oberstedt@eli-np.ro
} 


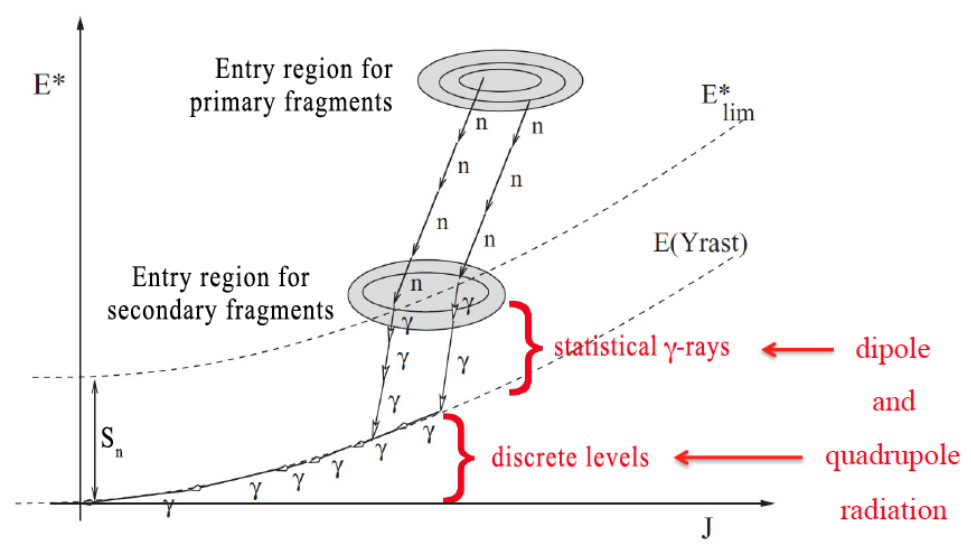

Figure 1. Illustration of the de-excitation process of fission fragments by the successive emission of neutrons and $\gamma$ rays. The change of excitation energy $\mathrm{E}^{*}$ with angular momentum $\mathbf{J}$ is indicated. Prompt fission $\gamma$ rays may be divided into statistical and discrete $\gamma$ rays, with a typical dipole and quadrupole character, respectively.

dipole transitions and carry away most of the remaining excitation energy of the fragments, while the latter stem mainly from electric quadrupole transitions along the Yrast line and carry away most of the angular momentum of the fragments [11]. This so-called prompt $\gamma$-ray emission is a fast process, which basically takes place within the first few nanoseconds after scission [12] and is followed by $\beta$ decay(s) and thereafter by delayed emission of neutrons and $\gamma$ rays towards the valley of $\beta$ stability. The average prompt fission $\gamma$-ray multiplicity, i.e. the average number of photons per fission, amounts typically to 8 [13], as corroborated by our measurements [1-6]. A schematic presentation of the de-excitation process of fission fragments is given in Fig. 1, in accordance with a picture from Ref. [14].

In this work now we have focused on the study of the de-excitation of fission fragments by prompt $\gamma$-ray emission with an attempt to deduce information about the relative contributions from radiation of different multipolarity. For that purpose we have chosen an experimental setup that allowed measuring angular correlations between fission fragments and the prompt $\gamma$-rays. Below we report on this recent measurement, present the observed angular distribution and give first preliminary results.

\section{Experimental setup and data treatment}

The recent measurement of PFGS from ${ }^{252} \mathrm{Cf}(\mathrm{sf})$ was performed with a 3 in. $\times 3$ in. (diameter $\times$ length) $\mathrm{LaBr}_{3}$ :Ce scintillation detector that was placed perpendicular to the plane of the sample along its symmetry axis. It was mounted inside a cylindrical twin Frisch-grid ionization chamber (FGIC) [15] in the center of a common cathode. Fission fragments from a thin ${ }^{252} \mathrm{Cf}$ sample are emitted to both sides of the cathode, ionizing the $\mathrm{P}-10$ counting gas, and being detected by means of the electrons moving towards the respective anodes. A so-called Frisch grid is placed in front of each anode in order to shield the latter from the influence of the ions, but it also allows determining the (polar) emission angle of the fragments (see Fig. 2 for a schematic view of the experimental setup). The FGIC provides the fission trigger for the coincident measurement of $\gamma$ rays, for which energy and time-of-flight (TOF) between sample and detector are recorded. The latter information is used to distinguish prompt $\gamma$ rays 


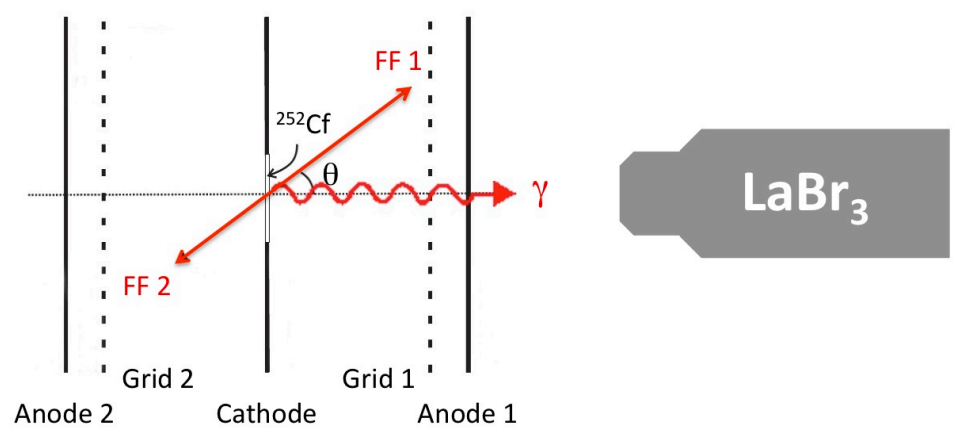

Figure 2. Sketch of the experimental setup used to measure angular correlations between fission fragments (FF 1 or FF 2) and prompt fission $\gamma$ rays. The fragments were detected with a cylindrical twin Frisch-grid ionization chamber (FGIC) on whose central cathode a ${ }^{252} \mathrm{Cf}$ sample was mounted. For the detection of the $\gamma$ rays a $\mathrm{LaBr}_{3}$ :Ce detector of size 3 in. $\times 3$ in. was employed that was placed along the symmetry axis perpendicular to the sample. The polar angle of the emitted fragments equivalent to the angle relative to the detected photons is denoted by $\theta$.

from other photons, emitted e.g. in prompt fission neutron induced reactions. The experimental techniques applied here are in principle the same as described in Refs. [1-6], where more details may be found.

The collected prompt fission $\gamma$ rays were sorted into energy spectra for $\cos \theta$ bins between 0 and 1 , with a width of 0.05 each. In order to obtain emission spectra, the response function of the detector has to be determined, which is usually done by means of Monte Carlo simulations of the actual setup with computer codes like e.g. Penelope [16] or Geant4 [17]. Thereafter the response function must be unfolded from the raw spectra. However, as a first step, we have chosen here a different, less time-consuming approach. Adding up all spectra gives an angular integrated raw spectrum that may be compared with others, obtained previously for the same fissioning system. Since they all turned out to exhibit a very similar appearance, we may benefit from the fact that similar raw spectra lead to similar emission spectra, provided that detector and setup are comparable [3]. This is the case here. From a previous measurement [1], for which the proper unfolding was carried out, both measured and emission spectra are known, whose ratio gives a transformation function (see Ref. [3] for details). Multiplying this function with the recently measured PFGS gives then the emission spectrum. The very good agreement between this one and a reference spectrum is apparent in Fig. 3, which justifies the procedure applied here. In the same way angular dependent emission spectra of prompt fission $\gamma$ rays were created for each bin, from which PFGS characteristics were determined. A detailed inspection of the obtained angular distribution is performed below.

\section{Angular distribution of prompt fission $\gamma$ rays}

As described in the previous chapter, emission spectra of prompt fission $\gamma$ rays were created for $\cos \theta$ bins, covering the polar angle range $0^{\circ}<\theta<90^{\circ}$. The integrated total multiplicity, i.e. the average number of emitted photons per fission, was determined to $\bar{M}_{\gamma}=8.16 \pm 0.29$, where the statistical uncertainty is 0.04 and the systematic one, mainly due to the transformation function, amounts 0.25 . This result is in good agreement with our last published value $\bar{M}_{\gamma}=8.29 \pm 0.13$ [2]. The angular distribution of radiation may be expressed according to 


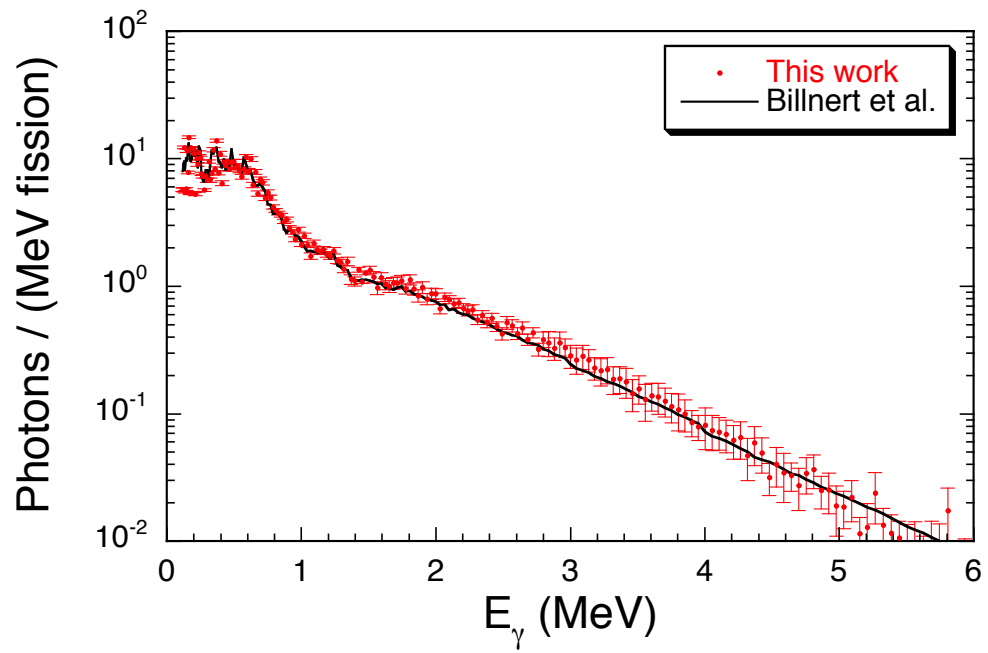

Figure 3. Measured PFGS spectrum for ${ }^{252} \mathrm{Cf}(\mathrm{sf})$ from this work, compared to a previously measured one from Ref. [1], exhibiting an excellent agreement.

$$
W(\theta)=A_{0}\left[1+\left\{A_{2} / A_{0}\right\} P_{2}(\cos \theta)+\left\{A_{4} / A_{0}\right\} P_{4}(\cos \theta)\right],
$$

where $P_{k}(\cos \theta)$ denote Legendre polynomials for $\mathrm{k}=2,4$ and $\theta$ is the emission angle of the fission fragment relative to the $\gamma$ ray direction, i.e. perpendicular to the cathode plane of the FGIC. Any possible attenuation coefficients are assumed to be 1 . The coefficients $\left\{A_{k} / A_{0}\right\}$ are determined experimentally and may be compared to theory for different types of radiation. For instance, $\left\{A_{2} / A_{0}\right\} \approx-0.3$ for pure dipole radiation and $\left\{A_{2} / A_{0}\right\} \approx 0.3$ for pure quadrupole radiation, while $\left\{A_{4} / A_{0}\right\}$ is close to zero [18]. Figure 4 shows the measured angular distribution as black dots, together with the result of a fit according to the expression given in Eq. 1 shown as line. It should be noted that the data close to both $\theta=0^{\circ}$ and $90^{\circ}$ suffers from considerable instrumental errors due to absorption and scattering, but they were taken into account anyway, since they did not influence much the fit result. The obtained coefficient is $\left\{A_{k} / A_{0}\right\}=0.13 \pm 0.03$, which corresponds to $72 \%$ quadrupole and $28 \%$ dipole radiation as shown as well in Fig. 4 (higher multipolarities are less probable and neglected here). This result is discussed below.

\section{Discussion and outlook}

Prior to this work, angular distributions of prompt $\gamma$ rays were measured in thermal-neutron induced fission of the target nuclei ${ }^{233} \mathrm{U},{ }^{235} \mathrm{U}$ and ${ }^{239} \mathrm{Pu}$ [19]. Comparison between all four measurements exhibits a good agreement, indicating a dominating contribution of quadrupole radiation, of which $\mathrm{E} 2$ is much more likely than M2. If this E2 radiation is assigned to $\gamma$ decays along the Yrast line (cf. Fig. 1), the corresponding multipolarity-dependent multiplicity would be $\bar{M}_{\gamma, L=2} \approx 6.0$, with a total average multiplicity of $\bar{M}_{\gamma}=8.29$ [2]. This implies an average angular momentum of $\mathrm{J} \approx 12 \hbar$, which is in rather good agreement with $\mathrm{J} \approx 10 \hbar$ reported earlier [20] and results of GEF calculations for different fission fragments [21]. The remaining dipole multiplicity would then be $\bar{M}_{\gamma, L=1} \approx 2.3$ for statistical $\gamma$ rays. 


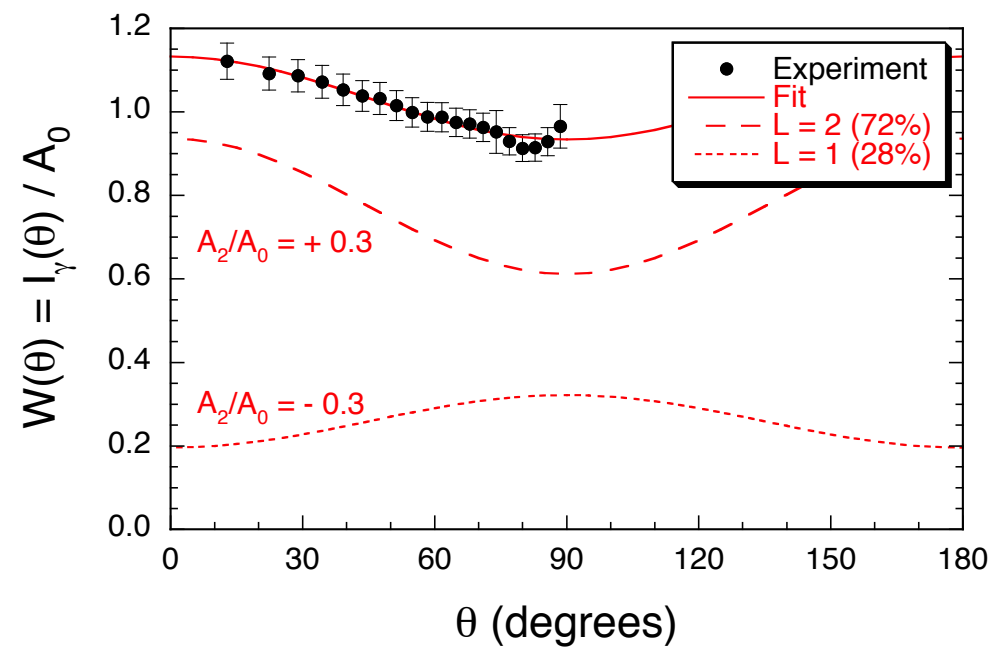

Figure 4. Measured angular distribution of prompt $\gamma$ rays from ${ }^{252} \mathrm{Cf}(\mathrm{sf})$, depicted as black dots. The error bars correspond to the sum of statistical and systematic uncertainties. The full drawn line represents the result of a fit according to Eq. 1, together with the deduced contributions of quadrupole $(L=2)$ and dipole $(L=1)$ radiation.

Calculations based on the Monte Carlo Hauser-Feshbach code FIFRELIN [14] have previously been quite successful in reproducing experimental results for PFGS characteristics as well as the distinct peak structure of the low-energy part of the energy spectra for ${ }^{235} \mathrm{U}\left(\mathrm{n}_{t h}, \mathrm{f}\right)$ [4] and ${ }^{252} \mathrm{Cf}(\mathrm{sf})$ [2]. Even multipolarity-dependent spectra were generated [22], which however seem to show a dominating contribution of $\mathrm{L}=1$ radiation. Apparently, this is in contrast with the results presented in this work, although a considerable part of radiation consists of so-called "experimental transitions", to which no multipolarity is assigned.

Hopefully this assignment will be able to be obtained with the help of experimental data, but in order to achieve that, the next step would have to be to extract energy spectra for different multipolarities. This should be possible by studying angular distributions of prompt fission $\gamma$ rays of different energies, which summarizes our future task in this context.

\section{Acknowledgments}

One of the authors (A. O.) acknowledges the support from the Extreme Light Infrastructure Nuclear Physics (ELI-NP) Phase II, a project co-financed by the Romanian Government and the European Union through the European Regional Development Fund - the Competitiveness Operational Programme (1/07.07.2016, COP, ID 1334), with which this work had been finalized.

\section{References}

[1] R. Billnert, F.-J. Hambsch, A. Oberstedt, and S. Oberstedt, Phys. Rev. C87, 024601 (2013).

[2] A. Oberstedt, R. Billnert, F.-J. Hambsch, and S. Oberstedt, Phys. Rev. C92, 014618 (2015).

[3] S. Oberstedt, A. Oberstedt, A. Gatera, A. Göök, A., F.-J. Hambsch, A. Moens, G. Sibbens, D. Vanleeuw, and M. Vidali, Phys. Rev. C93, 054603 (2016). 
[4] A. Oberstedt, T. Belgya, R. Billnert, R. Borcea, T. Bryś, W. Geerts, A. Göök, F.-J. Hambsch, Z. Kis, T. Martinez, S. Oberstedt, L. Szentmiklosi, K. Takács, and M. Vidali, Phys. Rev. C87, 051602(R) (2013).

[5] S. Oberstedt, R. Billnert, T. Belgya, T. Bryś, W. Geerts, C. Guerrero, F.-J. Hambsch, Z. Kis, A. Moens, A. Oberstedt, G. Sibbens, L. Szentmiklosi, D. Vanleeuw, and M. Vidali, Phys. Rev. C90, 024618 (2014).

[6] A. Gatera, T. Belgya, A. Göök, F.-J. Hambsch, M. Lebois, A. Oberstedt, S. Oberstedt, L. Qi, L. Szentmiklósi, G. Sibbens, and M. Vidali, accepted for publication in Phys. Rev. C.

[7] M. Lebois, J.N. Wilson, P. Halipré, A. Oberstedt, S. Oberstedt, P. Marini, C. Schmitt, S.J. Rose, S. Siem, M. Fallot, A. Porta, A.-A. Zakari, Phys. Rev. C92, 034618 (2015).

[8] J.-M. Laborie, R. Billnert, G. Bélier, A. Oberstedt, S. Oberstedt, and J. Taieb, submitted for publication in Phys. Rev. C.

[9] A. Oberstedt, R. Billnert, and S. Oberstedt,submitted for publication in Phys. Rev. C.

[10] C. Wagemans (editor), The Nuclear Fission Process, CRC Press Boca Raton (1991).

[11] A. Hotzel, P. Thirolf, Ch. Ender, D. Schwalm, M. Mutterer, P. Singer, M. Klemens, J. P. Theobald, M. Hesse, F. Gönnenwein, H. v. d. Ploeg, Z. Phys. A 356, 299 (1996).

[12] P. Talou, T. Kawano, I. Stetcu, J. P. Lestone, E. McKigney, and M. B. Chadwick, Phys. Rev. C94, 064613 (2016).

[13] R. Vandenbosch and J.R. Huizenga, Nuclear Fission, Academic Press New York and London (1973).

[14] O. Litaize and O. Serot, Phys. Rev. C82, 054616 (2010).

[15] C. Budtz-Jørgensen, H.-H. Knitter, Ch. Straede, F.-J. Hambsch and R. Vogt, Nucl. Instr. and Meth. A 258, 209 (1987).

[16] http://www.oecd-nea.org/tools/abstract/detail/nea-1525.

[17] S. Agostinelli and the Geant4 collaboration, Nucl. Inst. Meth. A 506 (2003) 250.

[18] E.S. Paul, UK Nuclear Physics Summer School, Bristol, 27. August - 6. September 2013, http://ns.ph.liv.ac.uk/ ajb/summerschool/files/ESP-Lecture4.pdf

[19] M.M. Hoffman, Phys. Rev. 133, B714 (1964).

[20] A. Bogachev, L. Krupa, O. Dorvaux, E. Kozulin, M. Itkis, M.-G. Porquet, A. Astier, D. Curien, I. Deloncle, G. Duchene, B.J.P. Gall, F. Hanappe, F. Khalfallah, M. Rousseau, L. Stuttge, N. Redon, and O. Stezowski, Eur. Phys. J. A 34, 23 (2007).

[21] K.-H. Schmidt, B. Jurado, C. Amouroux and C. Schmitt, Nuclear Data Sheets 131, 207 (2016).

[22] D. Regnier, "Contribution à l'étude des gammas prompts de fission", $\mathrm{PhD}$ thesis, Université de Grenoble, France (2013). 